\title{
A Case of Angiosarcoma Arising in Trunk of the Right Pulmonary Artery Clinically Simulating Pulmonary Thromboembolism
}

\author{
Yasuhiro Nakamura ${ }^{\mathrm{a}, \mathrm{e}}$, Toru Shimizu ${ }^{\mathrm{b}}$, Yoshihiro Fukumoto ${ }^{\mathrm{b}}$, Koichiro Sugimura ${ }^{\mathrm{b}}$, \\ Shigemi Ito ${ }^{c}$, Fumiyoshi Fujishima ${ }^{a}$, Mariko Oikawad ${ }^{\mathrm{d}}$, Mika Watanabe ${ }^{\mathrm{a}}$, \\ Hiroaki Shimokawa ${ }^{\text {b }}$ Hironobu Sasano ${ }^{\text {a }}$
}

\begin{abstract}
Angiosarcoma arising in the pulmonary artery is extremely rare. We herein report a case of angiosarcoma arising in the pulmonary artery trunk of 71 year-old woman. This case was clinically diagnosed as pulmonary thromboembolism but angiosarcoma of the pulmonary artery should be considered as the differential diagnosis of unusual clinical manifestations of pulmonary thromboembolism because of the extremely poor prognosis of the lesion.
\end{abstract}

Keywords: Angiosarcoma; Pulmonary artery; FDG-PET; Immunohistochemistry

\section{Introduction}

Angiosarcoma of pulmonary artery is very rare [1-15]. In addition, this tumor has been previously postulated to be indistinguishable from thromboembolism of the pulmonary artery $[13,15]$. We herein report a case of 71 year-old woman initially diagnosed as pulmonary thromboembolism and proven to be pulmonary angiosarcoma arising in the pulmonary artery trunk by autopsy.

Manuscript accepted for publication February 14, 2012

${ }^{\mathrm{a}}$ Department of Pathology, Tohoku University Graduate School of Medicine, Sendai, Japan

${ }^{b}$ Department of Cardiovascular medicine, Tohoku University Graduate School of Medicine, Sendai, Japan

${ }^{\mathrm{c}}$ Devision of Pathology, Miyagi Cancer Center, Natori, Japan

${ }^{\mathrm{d}}$ Division of Oral Pathology, Department of Oral Medicine and Surgery,

Tohoku University Graduate School of Dentistry, Sendai, Japan

${ }^{\mathrm{e}}$ Corresponding author: Yasuhiro Nakamura, Department of Pathology,

Tohoku University Graduate School of Medicine, 2-1 Seiryo-machi,

Aoba-ku, Sendai, 980- 8575, Japan.

Email: yasu-naka@patholo2.med.tohoku.ac.jp

doi: $10.4021 /$ wjon $467 \mathrm{w}$

\section{Case Report}

A 71-year-old woman with one month history of progressive shortness of breath, cough and atypical chest pain was hospitalized and diagnosed as pulmonary thromboembolism by computed tomography (CT) scan. She was then treated with heparin followed by warfarin, which mildly improved her symptoms but subsequent CT scan did not reveal any improvement. The patient was subsequently diagnosed as chronic thromboembolic pulmonary hypertension (CTEPH) and discharged. In 4 months later, she developed suddenonset of dyspnea, high fever and a large pericardial effusion on transthoratic echocardiography (TTE). The patient was readmitted to the intensive care unit of the hospital. Subseunet pericardiocenthesis revealed serosanginous pericardial effusion negative for infection and malignancy and she was subsequently referred to Tohoku University Hospital, Sendai, Japan.

Physical examination on admission revealed a loud P2 and a Grade III/IV systolic murmur, jugular vein dilatation and hepatomegaly. Her blood pressure was $90 / 60 \mathrm{mmHg}$, pulse rate 100 beats/min, body temperature $37.2^{\circ} \mathrm{C}, \mathrm{SpO} 2$ $98 \%$ with a $2 \mathrm{~L} / \mathrm{min} \mathrm{O} 2$ and she had a respiration rate of 20 breaths/min (NYHA III). The electrocardiogram demonstrataed sinus tachycardia (110 beats/min), P-wave pulmonale, $\mathrm{T}$ wave inversion on the right precordial leads, and incomplete right bundle block implying a pressure and volume overload of the right ventricle. Subsequent chest X-ray revealed cardiomegaly and bilateral pleural effusion with cardiothoracic ratio of $70 \%$. The laboratory investigation indicated anemia (hemoglobin $8.6 \mathrm{~g} / \mathrm{dl}$ ), heart failure (brain natriuteric peptide $183 \mathrm{pg} / \mathrm{ml}$ ), and suspected embolism (D-dimer $7.7 \mu \mathrm{g} / \mathrm{ml}$ ).

TTE revealed dilatation of the main pulmonary trunk and high systolic pressure gradient across the tricuspid valve of $49 \mathrm{mmHg}$ that is consistent with pulmonary hypertension. There was a thrombus-like mass in the right pulmonary artery and around the left atrium. CT scan similarly revealed a large mass in the proximal portion of right pulmonary artery and around the left atrium, but no abnormal mass detected in the lung and other organs (Fig. 1). However, lung scintigraphy demonstrated little visualization of the right lung on 


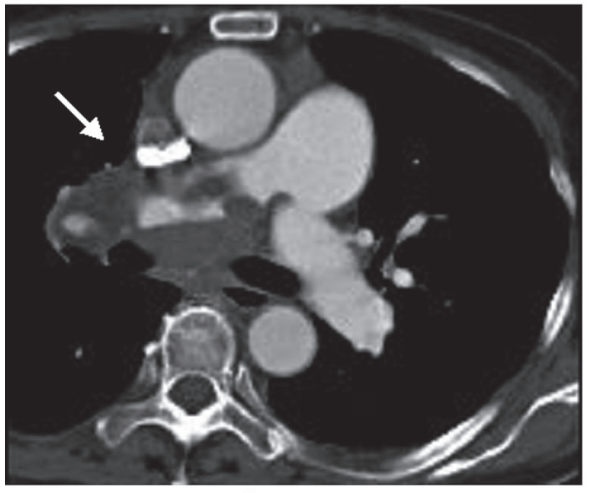

A

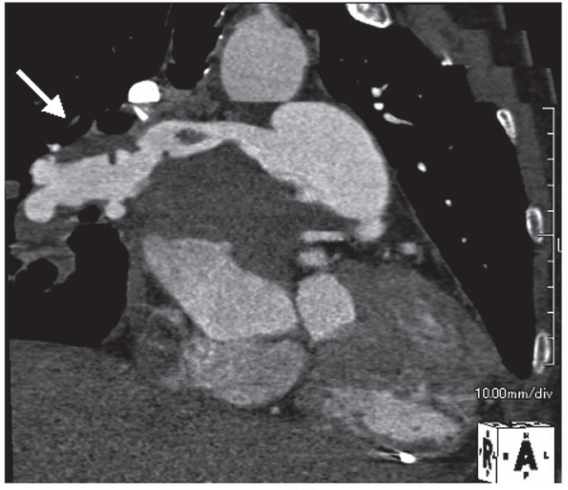

B

Figure 1. The image of Contrast-enhanced computed tomography scan. Early arterial phase showed massive filling defects in the proximal portion of right pulmonary artery and around the left atrium in axial (A) and sagittal $(B)$ views.

technetium-99m macroaggregated albumin (99mTc-MAA) perfusion scan. Ventilation scan with krypton-81m $(81 \mathrm{mKr})$ gas was normal (ventilation-perfusion mismatch). Cardiac catheterization demonstrated mean pulmonary artery pressure of $40 \mathrm{mmHg}$ that indicated pulmonary hypertension. Right pulmonary angiography demonstrated vascularized mass with a fistula from the right pulmonary artery to the left atrium (Fig. 2). To confirm this suspected malignant features and the extent of disease, 18-fluorodeoxyglucose positron emission tomography (FDG-PET) was performed. FDGPET revealed a large mass at the area of the right pulmonary artery and the bilateral atira with intense uptake of FDG. The maximum standard uptake value $\left(\mathrm{SUV}_{\max }\right)$ was 25.2. No other undue hypermetabolic lesions were noted elsewhere (Fig. 3).

On hospital day 24, a CT-guided transthoracic biopsy of the pericardial mass was performed. A histological diagnosis was a high-grade sarcoma, pleomorphic or spindle cell type and subsequent immunohistochemical findings consistent

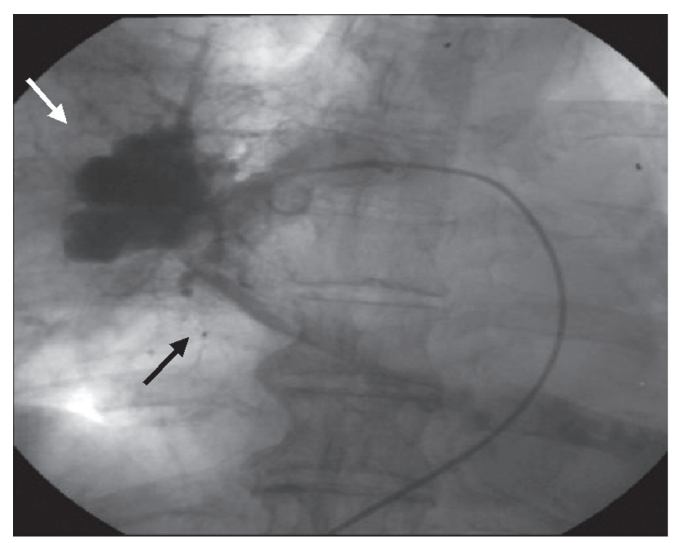

Figure 2. The image of right pulmonary angiography. Vascularized mass (white arrow) with a fistula from the right pulmonary artery to the left atrium (black arrow). with angiosarcoma. She consequently underwent chemotherapy with paclitaxel but on hospital day 55, the patient demised due to resistant right heart failure and consecutive respiratory failure during first-line chemotherapy.

An autopsy was performed. The tumor measured $20 \times 8$ $\mathrm{cm}$ in greatest diameter and occupied the upper pericardial space surrounding the pulmonary artery with direct invasion into both atriums and right lung (Fig. 4). The inner surface of the right pulmonary artery appeared irregular, and its second branched segment was completely occluded by the presence of tumor. Large coagulative thrombus was impacted in both atrioventricular outflows. Marked congestion and edema with focal hemorrhage were detected in bilateral lungs.

Histologically, the tumor was derived from the surface of the right pulmonary and was composed of pleomorphic cells harboring large and vesicular nuclei with prominent nucleoli and abundant eosinophilic cytoplasm (Fig. 4). Numerous mitoses and atypical mitoses were detected. Foci of hemorrhage and necrosis were also detected. Tumor cells focally formed vascular channels containing red blood cells. Immunohistochemically, the tumor cells were diffusely positive for vimentin, focally positive for CD31 and CD34 (Fig. 4) and negative for AE1/AE3, factor VIII-related antigen, $\alpha$-SMA, S-100, desmin, HHF-35, myoD1 and D2-40 (data not shown). MIB-index of tumor cells was approximately $15 \%$ (Fig. 4). The tumor was finally diagnosed as angiosarcoma originating in the trunk of the right pulmonary artery. Metastatic lesion was not detected.

\section{Discussion}

The incidence of pulmonary angiosarcoma is reported to be even only $3.6 \%$ among pulmonary artery sarcomas [2]. Huo et al reviewed 11 cases of angiosarcoma derived from pulmonary artery reported in the English literature [1] and reported as follows: the mean age of the 11 patients was 55 

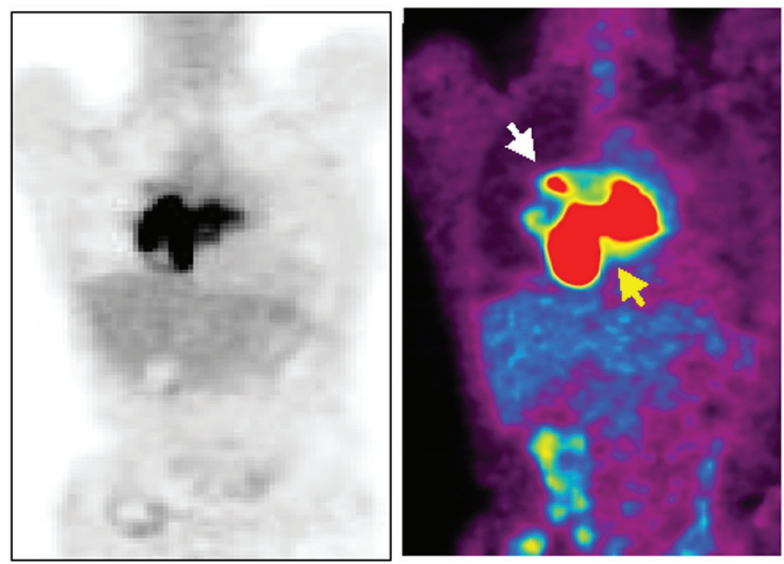

A

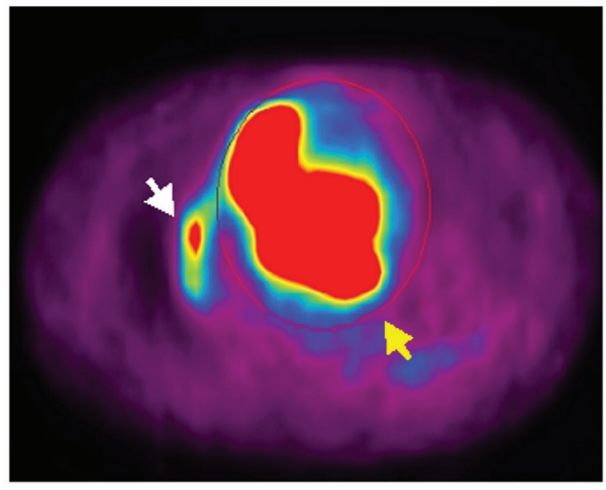

B

Figure 3. The image of maximum intensity projection image of 18-fluorodeoxyglucose positron emission tomography (FDG-PET). Increased uptake of FDG at the area of the right pulmonary artery (white arrow) and the bilateral atria (yellow arrow) in coronal (A) and axial $(B)$ views.

years (range, 38 - 69 years), and 5 were men and 2 were women among the 7 patients of known sex [1]. In the majority of cases $(n=8)$, the main trunk, right and/or left pulmonary artery were involved among the 10 cases of known tumor localization [1]. There was one case where only the right pulmonary artery was involved, and lung involvement was reported in 5 of 11 cases as in our case [1]. In addition, both the right and left atrium were involved by the tumor in our case, which has not been reported in any of the previously reported cases.

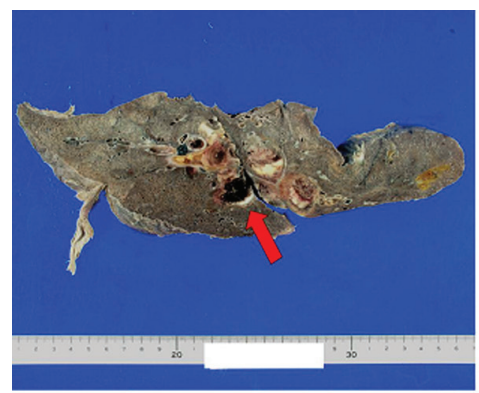

A

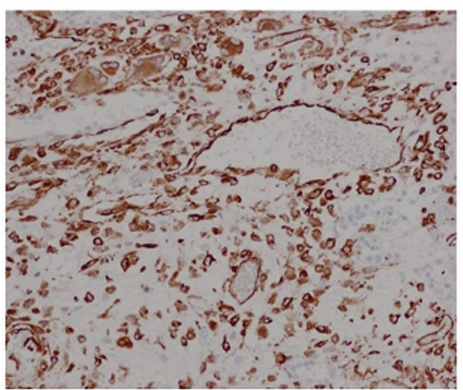

$\mathrm{D}$

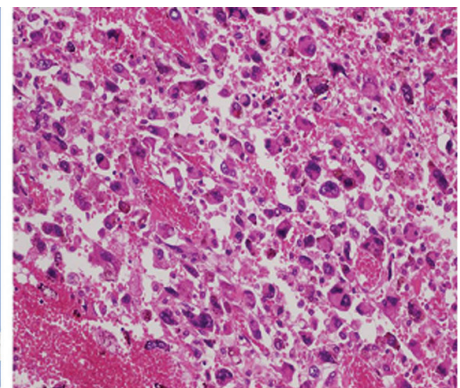

$\mathrm{B}$

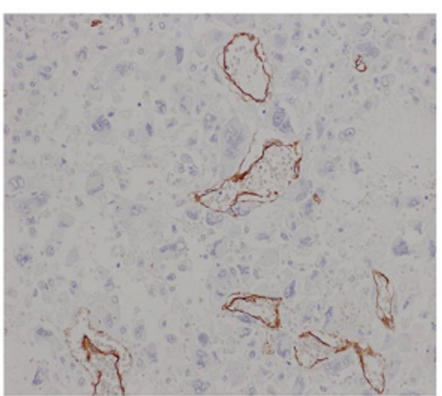

$\mathrm{E}$

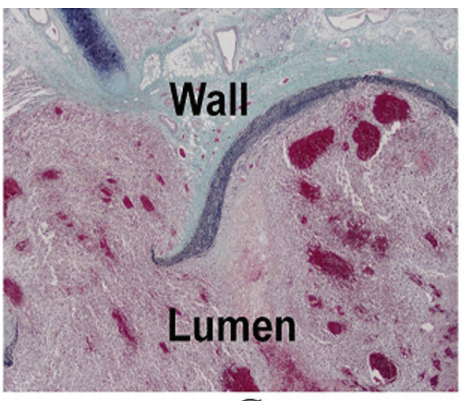

C

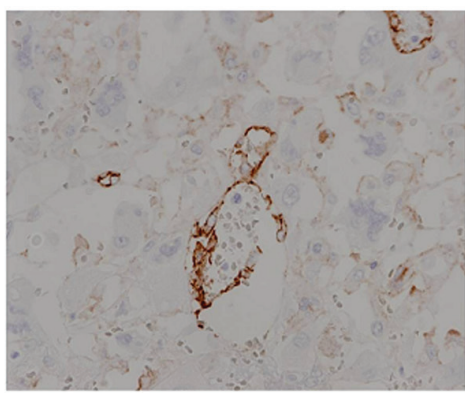

$\mathrm{F}$

Figure 4. A. Macroscopical finding showed that the right main trunk and its second branched segments of the pulmonary artery were completely occluded by the tumor and thrombus. A red arrow indicates the right pulmonary artery. B. Hematoxilin and eosin staining showed that the tumor cells were composed of pleomorphic cells which had large and vesicular nuclei with prominent nucleori and abundant eosinophilic cytoplasm. C. Elastica and masson staining showed the destruction of the wall of the right pulmonary artery by the tumor cells. 'Wall' indicates the wall of the right main trunk of the pulmonary artery, and 'Lumen' indicates its lumen. D. D, E and F: Immunohistochemical findings showed the tumor cells were diffusely positive for vimentin (D), focally positive for CD31 (E) and CD34 (F) in the vascular channel area. 
Echocardiography is a fundamental and useful diagnostic tool for pulmonary artery sarcoma. Contrast-enhanced CT scan or magnetic resonance imaging with gadolinium enhancement could be more beneficial for diagnosis of pulmonary artery sarcoma, which could provide the additional information regarding the size, location, attachment, and local extent of the tumor. Recently, FDG-PET has been reported to be useful for evaluation of the local invasion and distant metastasis of malignant cardiac tumors and for monitoring the response to therapy [16-19]. In addition, Ito et al reported FDG-PET can distinguish pulmonary artery sarcoma from venous pulmonary thromboembolism based on the FDG uptake with $\mathrm{SUV}_{\text {max }}(7.63 \pm 2.21$ vs. $2.31 \pm 0.41)$ [18]. In our case, the high FDG uptake with $\mathrm{SUV}_{\text {max }}$ of 25.2 has highly suspected pulmonary artery sarcoma, but not thromboembolism.

The tumor in our case was diagnosed as angiosarcoma at autopsy. The histological features of our present case demonstrated a high-grade spindle and pleomorphic cell with vascular channels. Immunohistochemically, the tumor cells were diffusely positive for vimentin, focally positive for CD31 and CD34 in our case, which are consistent with immunohistochemical features of angiosarcoma [14]. While cytokeratin was negative for the tumor in our case, previous studies demonstrated cytokeratin immunoreactivity in pulmonary angiosarcoma $[4,14]$.

The most common presenting symptoms are known to be non-specific, including cough, dyspnea, chest pain and intermittent hemoptysis $[1,13]$. Therefore, several previous cases were initially diagnosed as pulmonary thromboembolism [8, 13, 15]. It has been postulated that pulmonary artery angiosarcoma should be included in the differential diagnosis of acute or chronic pulmonary thromboembolism at initial diagnosis, since the prognosis of pulmonary artery sarcoma in considered extremely poor. However, Engelke et al. postulated that pulmonary thromboembolism caused by primary pulmonary malignancy is difficult for therapy since thrombolysis or therapeutic anticoagulation are usually contraindicated in patients with underlying pulmonary malignancy to prevent serious bleeding complications [15]. In addition, biopsy could contribute greatly to the differential diagnosis between primary parenchymal and pulmonary arterial sarcomatous tissues [15]. It is postulated that pulmonary artery sarcoma is considered a disease of unilateral pulmonary artery involvement, whereas pulmonary embolism seems comparatively to be a disease of bilateral pulmonary artery involvement as seen in our case [20]. Therefore, in our pesent case, pulmonary artery angiosarcoma should have been included at the initial diagnosis.

\section{References}

1. Huo L, Lai S, Gladish G, Czerniak BA, Moran CA. Pul- monary artery angiosarcoma: a clinicopathologic and radiological correlation. Ann Diagn Pathol. 2005;9(4):209214.

2. Nonomura A, Kurumaya H, Kono N, Nakanuma Y, Ohta G, Terahata S, Matsubara F, et al. Primary pulmonary artery sarcoma. Report of two autopsy cases studied by immunohistochemistry and electron microscopy, and review of 110 cases reported in the literature. Acta Pathol Jpn. 1988;38(7):883-896.

3. Burke AP, Virmani R. Sarcomas of the great vessels. A clinicopathologic study. Cancer. 1993;71(5):1761-1773.

4. Goldblum JR, Rice TW. Epithelioid angiosarcoma of the pulmonary artery. Hum Pathol. 1995;26(11):1275-1277.

5. Zerkowski HR, Hofmann HS, Gybels I, Knolle J. Primary sarcoma of pulmonary artery and valve: multimodality treatment by chemotherapy and homograft replacement. J Thorac Cardiovasc Surg. 1996;112(4):1122-1124.

6. Kacl GM, Bruder E, Pfammatter T, Follath F, Salomon F, Debatin JF. Primary angiosarcoma of the pulmonary arteries: dynamic contrast-enhanced MRI. J Comput Assist Tomogr. 1998;22(5):687-691.

7. Govender D, Pillay SV. Right pulmonary artery sarcoma. Pathology. 2001;33(2):243-245.

8. Tschirch FT, Del Grande F, Marincek B, Huisman TA. Angiosarcoma of the pulmonary trunk mimicking pulmonary thromboembolic disease. A case report. Acta radiol. 2003;44(5):504-507.

9. Totaro M, Miraldi F, Ghiribelli C, Biscosi C. Cardiac angiosarcoma arising from pulmonary artery: endovascular treatment. Ann Thorac Surg. 2004;78(4):1468-1470.

10. Garate ML, Ochoa JP, Stampone G, Gurfinkel EP. [Endovascular diagnosis and palliative treatment of a pulmonary artery angiosarcoma]. Rev Esp Cardiol. 2011;64(12):1216-1217.

11. Said SM, Sundt TM, 3rd, Garces YI, Wigle DA. 5-year survival after multiple repeat metastasectomy for pulmonary artery angiosarcoma. Ann Thorac Surg. 2011;91(4):e49-51.

12. Mendiz O, Lev G, Valdivieso L, Fava C, Gallucci E, Baldessari E, Favaloro R. Lifesaving kissing stent for pulmonary trunk stenosis due to primary angiosarcoma. Ann Vasc Surg. 2010;24(8):1135 e1139-1112.

13. Kim JB, Kim SH, Lim SY, Roh SY, Cho GY, Song HJ, Park S, et al. Primary angiosarcoma of the pulmonary trunk mimicking pulmonary thromboembolism. Echocardiography. 2010;27(2):E23-26.

14. Huo L, Moran CA, Fuller GN, Gladish G, Suster S. Pulmonary artery sarcoma: a clinicopathologic and immunohistochemical study of 12 cases. Am J Clin Pathol. 2006;125(3):419-424.

15. Engelke C, Riedel M, Rummeny EJ, Marten K. Pulmonary haemangiosarcoma with main pulmonary artery thrombosis imitating subacute pulmonary embolism with infarction. Br J Radiol. 2004;77(919):623-625. 
16. Ote EL, Oriuchi N, Miyashita G, Paudyal B, Ishikita T, Arisaka Y, Higuchi T, et al. Pulmonary artery intimal sarcoma: the role of (1)F-fluorodeoxyglucose positron emission tomography in monitoring response to treatment. Jpn J Radiol. 2011;29(4):279-282.

17. Chun IK, Eo JS, Paeng JC, Kim DW, Chung JK, Lee DS. Pulmonary artery sarcoma detected on F-18 FDG PET/ $\mathrm{CT}$ as origin of multiple spinal metastases. Clin Nucl Med. 2011;36(8):e87-89.

18. Ito K, Kubota K, Morooka M, Shida Y, Hasuo K, Endo H,
Matsuda H. Diagnostic usefulness of 18F-FDG PET/CT in the differentiation of pulmonary artery sarcoma and pulmonary embolism. Ann Nucl Med. 2009;23(7):671-676.

19. Farsad M, Pernter P, Triani A, Osele L, Wiedermann CJ. Thromboembolism in pulmonary artery sarcoma. Clin Nucl Med. 2009;34(4):239-240.

20. Yi CA, Lee KS, Choe YH, Han D, Kwon OJ, Kim S. Computed tomography in pulmonary artery sarcoma: distinguishing features from pulmonary embolic disease. J Comput Assist Tomogr. 2004;28(1):34-39. 